\title{
An Economic Comparison of Treatment Strategies with Anakinra in Systemic Juvenile Idiopathic Arthritis (sJIA)
}

\author{
Ash Bullement (iD) \\ Emma S Knowles (D) \\ Merel Langenfeld ${ }^{2}$ \\ Gil Reynolds Diogo ${ }^{3}$ \\ Jameel Nazir ${ }^{4}$ \\ Daniel Eriksson (iD) ${ }^{4}$ \\ 'Delta Hat, Nottingham, UK; ${ }^{2}$ Sobi, \\ Woluwe-Saint-Lambert, Belgium; ${ }^{3}$ Sobi, \\ Cambridge, UK; ${ }^{4}$ Sobi, Stockholm, \\ Sweden
}

Introduction: Systemic juvenile idiopathic arthritis (sJIA) is a rare, complex autoinflammatory disease with substantial morbidity, often characterized by fever, rash, and muscle pain, amongst other symptoms. Biologic agents, such as anakinra, have been successfully used to treat patients internationally, but their usage in some regions is limited to patients that have failed to achieve clinically inactive disease with corticosteroids and conventional synthetic disease-modifying anti-rheumatic drugs (csDMARDs). Use of anakinra early in the disease course leads to better clinical outcomes; however, longer-term costs for this treatment strategy have not been established. This study compares the economic implications of first-line versus later-line availability of anakinra for patients with sJIA.

Methods: Data for patients treated with first-line anakinra were identified from a singlecenter, prospective study and compared to a combination of published trial and economic evaluation information to facilitate a comparison to later-line anakinra (ie, following corticosteroids + csDMARDs). Costs were estimated for product acquisition and medical resource utilization (MRU), including planned outpatient visits and unplanned hospital admissions. Total costs over a 5-year horizon were compared.

Results: Total 5-year product acquisition cost for the first-line anakinra strategy was $€ 24,021$, and for later-line anakinra was $€ 20,471$. The corresponding MRU costs were $€ 19,197$ (first-line) versus $€ 25,425$ (later-line). Overall 5-year costs (product acquisition and MRU) were lower for the first-line strategy (€43,218 versus €45,896).

Conclusion: The use of anakinra for patients with sJIA in the first-line setting is efficacious to induce and sustain inactive disease, and the findings of this study show that this treatment strategy leads to cost savings through reduced medical expenditure.

Keywords: Still's disease, systemic juvenile idiopathic arthritis, anakinra, economic comparison, treatment strategies, first line

\section{Plain Language Summary}

Anakinra, a treatment for patients with systemic juvenile idiopathic arthritis (sJIA), has been used in patients with sJIA for over a decade. Different regions treat with anakinra at different stages in the treatment pathway. In some regions, anakinra is used as a first-line treatment option, whereas in others, its use is reserved for patients who fail to respond to treatment with non-steroidal anti-inflammatory drugs (NSAIDs) and conventional synthetic diseasemodifying antirheumatic drugs (csDMARDs), or, in other words, "later-line" use of anakinra. This study presents the first economic comparison of different treatment strategies in sJIA. We identified data from a range of different sources and estimated the costs of total drug and medical resource use associated with each alternative treatment regimen. We then compared
Correspondence: Daniel Eriksson Email daniel.eriksson@sobi.com 
the total costs accrued over a five-year period. The results of our analysis show first-line anakinra treatment is associated with cost savings versus later-line use, when considered in the context of the full cost implications of patient management.

\section{Introduction}

Systemic juvenile idiopathic arthritis (sJIA) is a rare, complex autoinflammatory disease, with an incidence of $0.5-$ 0.9 per 100,000 children. $^{1-4}$ It is associated with substantial morbidity, and is usually characterized by spiking fever, rash, muscle pain, arthritic symptoms, in addition to liver and spleen enlargement. ${ }^{5-7}$ With an average age of diagnosis between 3 and 5 years, children with sJIA have reduced quality of life, caused by impaired physical and social functioning, which is especially detrimental in developing years. ${ }^{8,9}$

While sJIA is often considered a subtype of juvenile idiopathic arthritis (JIA), its systemic nature differentiates it from other, more common rheumatic diseases in several aspects. It is understood to be a polygenic autoinflammatory disease characterized by an excessive activation of the innate immune system and secretion of interleukin-1 (IL$1),-6$, and -18 . It is therefore different to other forms of JIA that are considered to be autoimmune where the pathology is predominantly driven by the adaptive immune system. ${ }^{10}$ sJIA is also described as the most severe and potentially life-threatening disease in the group of JIA. ${ }^{10}$ More recently, sJIA and adult-onset Still's disease (AOSD) have been described as the same disease entity with different ages of onset. ${ }^{11}$

Owing to its systemic nature, sJIA is one of very few rheumatic diseases associated with the development of macrophage activation syndrome (MAS) - a potentiallyfatal complication, recognized as the most frequent lifethreatening complication of Still's disease. ${ }^{12,13}$ Therefore, in addition to substantial morbidity, sub-optimal management of sJIA is associated with an increased risk of mortality.

Traditionally, the management of sJIA was concerned primarily with reducing the risk of long-term complications associated with the disease (including issues related to long-term steroid use, and the requirement for articular surgery), as opposed to achieving a state of clinicallyinactive disease (CID, or "remission" - that is, an absence of disease-related symptoms and normalization of laboratory markers). This was a direct consequence of the lack of efficacious treatments that had been specifically studied within sJIA populations, and so clinical practice historically comprised the use of unlicensed treatments that had been studied in other rheumatic diseases. These unlicensed treatment options for sJIA include nonsteroidal anti-inflammatory drugs (NSAIDs), corticosteroids, and conventional-synthetic disease-modifying antirheumatic drugs (csDMARDs). Moreover, the only randomized, placebo-controlled trial of methotrexate (csDMARD) in patients with sJIA did not demonstrate a statistically significant improvement in the systemic features of the disease. ${ }^{14}$

While traditional therapies were able to improve symptoms, their mechanistic properties did not allow for patients to achieve a state of CID. Following increased understanding of the role of IL-1/IL-6 in sJIA, biologic DMARDs (bDMARDs) were studied in sJIA populations in a number of clinician-led studies. ${ }^{14-17}$ Anakinra (Kineret $^{\circledR}$, Sobi), an IL-1 receptor antagonist, has been used to treat patients with sJIA for over a decade across Europe, ${ }^{18}$ and was licensed for the treatment of Still's disease (including sJIA) in April 2018. ${ }^{19,20}$ With the availability of bDMARDs targeting IL-1/IL-6, treatment goals shifted from management of symptoms to achieving CID.

Clinical studies have shown that it is possible to achieve CID with bDMARDs, though outcomes are particularly impressive for patients treated with anakinra shortly after diagnosis. ${ }^{21}$ Cytokine blockade early in the course of Still's disease may allow patients to achieve remission before the development of chronic, destructive, and often therapy-resistant arthritis. ${ }^{20-25}$

A number of clinical guidelines have been updated to reflect the expected benefits associated with earlier use of bDMARDs, acknowledging more recent clinical studies. $^{11,26,27}$ Cohorts of sJIA patients in the Netherlands and Italy have been treated with first-line anakinra, and a majority of patients were shown to achieve CID, many of whom were able to discontinue therapy in the longer term. ${ }^{21,25,28,29}$ Earlier use of anakinra has been shown to be associated with clinical benefits; however, it is important to note that this strategy may have an impact on the overall cost of managing patients. To establish its cost-effectiveness, a comparison of hypothetical treatment pathways (ie, use of anakinra early versus later in the treatment pathway) is required.

To our knowledge, no comparison of treatment strategies in sJIA from an economic perspective have been previously conducted. This study aimed to formally compare alternative treatment strategies with anakinra (where it is available in a first-line versus later line setting) in the 
management of sJIA to illustrate the differences in expected costs.

\section{Materials and Methods Strategies to be Compared}

This study considers a comparison of two treatment strategies:

1. First-line anakinra: This strategy is concerned with the use of anakinra as soon as possible after diagnosis, theoretically prior to the use of any active treatment for sJIA (including corticosteroids). This strategy is aligned with the latest Single Hub and Access point for pediatric Rheumatology in Europe (SHARE) treatment guidelines, ${ }^{11}$ the Dutch Pediatrics Association guidelines (Nederlandse Vereniging voor Kindergeneeskunde, NVK), ${ }^{26}$ and a number of recent studies. ${ }^{21,25,28,29}$

2. Later-line anakinra: This strategy involves the use of anakinra after the use of NSAIDs, corticosteroids, and at least one csDMARD (such as methotrexate). Use of anakinra in this setting is aligned with the use of anakinra in earlier published studies (such as ANAJIS ${ }^{15}$ ) and is representative of current practice in the UK (per NICE TA238 and TA685 guidance, and a National Health Service clinical commissioning policy). ${ }^{30-32}$

\section{Evidence Base}

Most countries treat sJIA patients based on a national policy (including the UK and the Netherlands). ${ }^{26,30,31}$ Therefore, it is unlikely that a head-to-head comparison of alternative treatment strategies in the same country would be possible (eg, comparing strategies where anakinra is available in the first-line setting, versus only being available in a later-line setting). Accordingly, data to inform both treatment strategies were sought from the literature, with the expectation that supporting studies are likely to have been conducted in different geographies. Where literature was unavailable, information from published reports (eg, NICE guidance) were considered, else assumptions were made where necessary (which are described where applicable throughout).

For patients treated using a first-line anakinra strategy, data regarding the duration of treatment and proportion of patients that achieve CID were taken from ter Haar et al. ${ }^{21}$ This study reports on a population of $n=42$ patients with
sJIA treated with anakinra monotherapy according to a treat-to-target strategy (before the use of corticosteroids), and followed for a median of 5.8 years. ${ }^{21}$ At 1 year, 76\% of patients (30/42) had CID and 52\% (22/42) had CID off medication. $^{21}$ For those that were followed up to 5 years, 96\% (24/25) had CID, and 72\% (18/25) had CID off medication. $^{21}$

In contrast to a first-line treatment strategy, there is no equivalent, comprehensive study available to consider the costs and outcomes for a later-line anakinra treatment strategy. However, tocilizumab (RoActemra ${ }^{\circledR}$, Roche, an IL-6 inhibitor) was previously assessed by the National Institute for Health and Care Excellence (NICE) in this setting (TA238), which is broadly aligned with the use of anakinra for sJIA in a later-line setting. ${ }^{30}$ Therefore, data and assumptions used to inform NICE TA238 were used to produce estimated costs for the later-line anakinra setting, supported with additional information from the literature where needed.

Patients managed with a later-line anakinra strategy were assumed to be treated with NSAIDs, corticosteroids, and a csDMARD (methotrexate) prior to the initiation of bDMARD therapy. Approximately a quarter of patients $(25.5 \%)$ were assumed to have monocyclic disease course $^{33}$ for which it was assumed possible for patients to achieve CID prior to the initiation of bDMARDs. The remaining patients were assumed to have chronic disease course. For NSAIDs + corticosteroids, it was assumed that approximately $30 \%$ of monocyclic patients would achieve remission within 6 weeks, and for methotrexate, $20 \%$ would achieve remission within 24 weeks (based on the Nordström et al study in AOSD, where it was assumed all patients that achieved "remission" had monocyclic disease course $^{17}$ ).

Approximately $50 \%$ of patients (with either monocyclic or chronic disease course) were assumed to achieve CID with the first bDMARD used based on the anakinra arm of the Nordström et al. study. ${ }^{17}$ In the base-case analysis, it was assumed all patients would currently receive tocilizumab as the first choice of biologic therapy after csDMARDs (based on its availability as a licensed treatment option for sJIA since August 2011). After failure on tocilizumab, patients would switch to anakinra. Given the lack of head-to-head comparisons between bDMARDs in sJIA patients, both products were assumed to have the same efficacy.

Discontinuation rates were calibrated based on the probabilities of achieving CID, background mortality, 
and the expectation that nearly all patients will have discontinued NSAIDs + corticosteroids and methotrexate after 6 and 16 weeks, respectively. To do this, a probability of discontinuation per week was estimated assuming 95\% would discontinue in these time periods. For bDMARDs, data from NICE TA238 (tocilizumab for sJIA) were taken, in which $12.6 \%$ of patients were assumed to discontinue bDMARD treatment per year. ${ }^{30}$ It was also assumed that some patients would continue treatment with bDMARDs with CID. In the base-case analysis, this was assumed to be $50 \%$.

\section{Analysis Methodology}

A micro-costing analysis was undertaken to estimate the total costs related to product acquisition and medical resource use (MRU). Product acquisition costs were based on estimated durations of treatment from ter Haar et al (first-line) and TA238 (later-line). ${ }^{21,30}$ Unit costs were taken from Dutch reference cost sources. ${ }^{34}$

MRU was assumed to be related to whether or not patients still had CID. In the base-case analysis, four cost items were considered: outpatient rheumatology appointments, outpatient hematology appointments, general practitioner (GP) visits, and unplanned hospitalizations. Resource use frequencies were sourced from NICE TA238, with a revision to the expected number of outpatient rheumatology appointments per year to align with approximately one appointment per month. ${ }^{30} \mathrm{~A}$ table of parameters included in the analysis is provided in Table 1.

The analysis takes the hypothetical perspective of a European cohort of patients, given that no single location is expected to consider a blend of both treatment strategies. Drug and MRU costs are presented in Euros over a 5-year horizon, in line with standard budget impact analysis convention, which are then combined to calculate the overall 5-year costs associated with each treatment strategy. Sensitivity analyses including additional test costs, costs associated with MAS and long-term complications of treatment were considered to explore key areas of uncertainty.

\section{Results}

\section{Headline Analysis Results}

The results of the base-case analysis show that the total 5 -year drug costs for the first-line anakinra strategy were $€ 24,021$, versus $€ 20,471$ for later-line anakinra; and the corresponding MRU costs were $€ 19,197$ (first-line) versus
$€ 25,425$ (later-line) (Figure 1). Therefore, the overall 5 -year costs (drug acquisition and MRU) were lower for the first-line strategy ( $€ 43,218$ versus $€ 45,896)$, demonstrating that first-line anakinra is cost saving $(-€ 2,677)$ relative to a later-line strategy. In other words, while firstline anakinra was associated with increased spend related to drug acquisition, it led to cost savings associated with reduced medical resource use expenditure including, for example, a reduction in hospital stays.

\section{Sensitivity Analyses}

A number of sensitivity analyses were conducted to further explore the likely differences in the costs associated with each treatment strategy.

The MRU items included in the base-case analysis were selected on the basis of these costs representing the majority of direct healthcare costs incurred. However, additional costs may be incurred in a real-world setting. A sensitivity analysis was conducted to include the costs of C-reactive protein and creatinine clearance tests as well as public transport for outpatient consultations. By including both of these transport-related additional costs, the savings associated with first-line anakinra use increased further to $-€ 2714$.

Patients with active disease are subject to a risk of developing MAS, yet these costs were not captured in the base-case analysis. To explore the impact of including MAS-related costs in the analysis, we considered a sensitivity analysis wherein patients with active disease had an average of 0.0525 MAS events per year (based on a study by Grom et $\mathrm{al}^{36}$ ). The cost of resolving MAS was assumed to be equivalent to a 14-day stay in intensive care, including diagnostics and medication. Including MAS-related costs, the base-case cost savings increased to $-€ 3225$.

It is also understood that patients who receive prolonged treatment with corticosteroids and/or csDMARDs (such as methotrexate) may develop long-term complications related to treatment. ${ }^{37}$ In a sensitivity analysis, we considered an additional cost of a hospitalization each year for $18 \%$ of patients (ie, the difference in patients not in remission at 5 years, see Table 1) treated with a later-line strategy after 5 years for an additional 5 years, as a crude exploration of the potential impact of including longerterm complications on results. By including this cost, the cost savings increased to $-€ 3249$. 
Table I Economic Analysis Input Parameters

\begin{tabular}{|l|l|l|}
\hline Input & Value & \multirow{2}{*}{ Reference } \\
\hline $\begin{array}{l}\text { Clinical parameters (first-line anakinra) } \\
\text { These parameters are used to establish which proportion of patients have CA } \\
\text { patients managed with first-line anakinra. }\end{array}$ & \multirow{2}{*}{ ter Haar et al. ${ }^{21}$} \\
\cline { 1 - 2 } CID at I year & $76 \%$ & \\
\cline { 1 - 2 } CID at 3 years & $94 \%$ & \\
\cline { 1 - 2 } CID at 5 years & $96 \%$ & \\
\cline { 1 - 2 } CID off treatment at I year & $52 \%$ & \\
\cline { 1 - 2 } CID off treatment at 3 years & $71 \%$ & $72 \%$ \\
\cline { 1 - 2 } CID off treatment at 5 years & &
\end{tabular}

Clinical parameters (later-line anakinra)

These parameters are used to establish which proportion of patients have CAD versus CID over the time frame of the economic analysis, for patients managed with later-line anakinra. A multitude of sources is used for this treatment strategy, owing to a lack of data available specifically reporting on the experience of sJIA patients managed with later-line anakinra following initial treatment with NSAIDs and csDMARDs.

\begin{tabular}{|c|c|c|}
\hline Monocyclic course & $25.5 \%$ & Grevich and Shenoi ${ }^{33}$ \\
\hline CID w/ corticosteroids (monocyclic) & $30 \%$ (in 6 weeks) & Assumption \\
\hline CID w/ methotrexate (monocyclic) & $20 \%$ (in 24 weeks) & Assumption based on Nordström et al. ${ }^{17}$ \\
\hline CID w/ corticosteroids (chronic) & $0 \%$ & Assumption \\
\hline CID w/ methotrexate (chronic) & $0 \%$ & Assumption \\
\hline CID w/ bDMARDs & $50 \%$ (in 24 weeks) & Assumption based on Nordström et al. ${ }^{17}$ \\
\hline $\begin{array}{l}\text { Discontinuation for NSAIDs + } \\
\text { corticosteroids }\end{array}$ & $95 \%$ off by 6 weeks & Assumption \\
\hline $\begin{array}{l}\text { Discontinuation for csDMARD } \pm \\
\text { NSAIDs + corticosteroids }\end{array}$ & $95 \%$ off by 16 weeks & Assumption \\
\hline Discontinuation for bDMARDs & $12.6 \%$ per year & NICE TA238 30 \\
\hline Proportion on bDMARDs with CID & $50 \%$ & Assumption \\
\hline CID at 5 years & $78 \%$ & Calculation based on input parameters above \\
\hline Difference in CID at 5 years & $18 \%$ & \\
\hline
\end{tabular}

Drug costs per week

These parameters are used to inform drug acquisition cost results. These costs refer to the estimated cost per week.

\begin{tabular}{|c|c|c|}
\hline NSAIDs + corticosteroids & $€ 0.82$ & \multirow[t]{4}{*}{ Z-Index B.V. ${ }^{35}$} \\
\hline csDMARD & $€ 18.88$ & \\
\hline Anakinra & $€ 225.40$ & \\
\hline Tocilizumab & $€ 275.68$ & \\
\hline
\end{tabular}

Annual medical resource use

These parameters are used to estimate the medical resource utilization associated with patients that have CAD versus CID, noting that in general patients with CAD require additional resource. 
Table I (Continued).

\begin{tabular}{|c|c|c|}
\hline Input & Value & Reference \\
\hline Rheumatology (CAD) & 12.0 & \multirow{4}{*}{$\begin{array}{l}\text { NICE TA238. }{ }^{30} \text { For rheumatology, increased from } 10 \text { reported in NICE TA238 to } 12 \\
\text { reflecting an average of I appointment per month }\end{array}$} \\
\hline Hematology (CAD) & 12.0 & \\
\hline GP $(C A D)$ & 20.8 & \\
\hline Hospitalization (CAD) & 22.1 & \\
\hline Rheumatology (CID) & 4.0 & Assumed I appointment every 3 months \\
\hline GP (CID, on treatment) & 3.5 & NICE TA238 30 \\
\hline GP (CID, off treatment) & 0.0 & Assumption \\
\hline \multicolumn{3}{|c|}{$\begin{array}{l}\text { Unit costs for medical resource use } \\
\text { These parameters are used to assign costs based on the expected frequency of use (separated by CAD versus CID, as shown in the preceding } \\
\text { rows). }\end{array}$} \\
\hline Rheumatology or hematology & $€ 101.00$ & iMTA costing tool: ${ }^{34}$ Outpatient visit, pediatrics \\
\hline GP & $€ 33.00$ & iMTA costing tool: ${ }^{34}$ General practitioner, standard consultation \\
\hline Hospitalization & $€ 642.00$ & iMTA costing tool: ${ }^{34}$ Nursing day, academic hospital \\
\hline
\end{tabular}

Abbreviations: bDMARD, biologic disease-modifying anti-rheumatic drug; csDMARD, conventional synthetic disease-modifying anti-rheumatic drug; CAD, clinically-active disease; CID, clinically-inactive disease; GP, General practitioner; iMTA, Institute for Medical Technology Assessment; NICE, National Institute for Health and Care Excellence; NSAID, non-steroidal anti-inflammatory drug; TA, technology appraisal.

\section{Discussion}

This study demonstrates the economic implications of alternative treatment strategies (first-line versus laterline) with anakinra (in other words, either used in the first-line setting, or in a refractory setting after failure to achieve CID with NSAID, corticosteroid, and csDMARD treatment [eg, methotrexate]). The results of the analysis demonstrate that first-line anakinra is

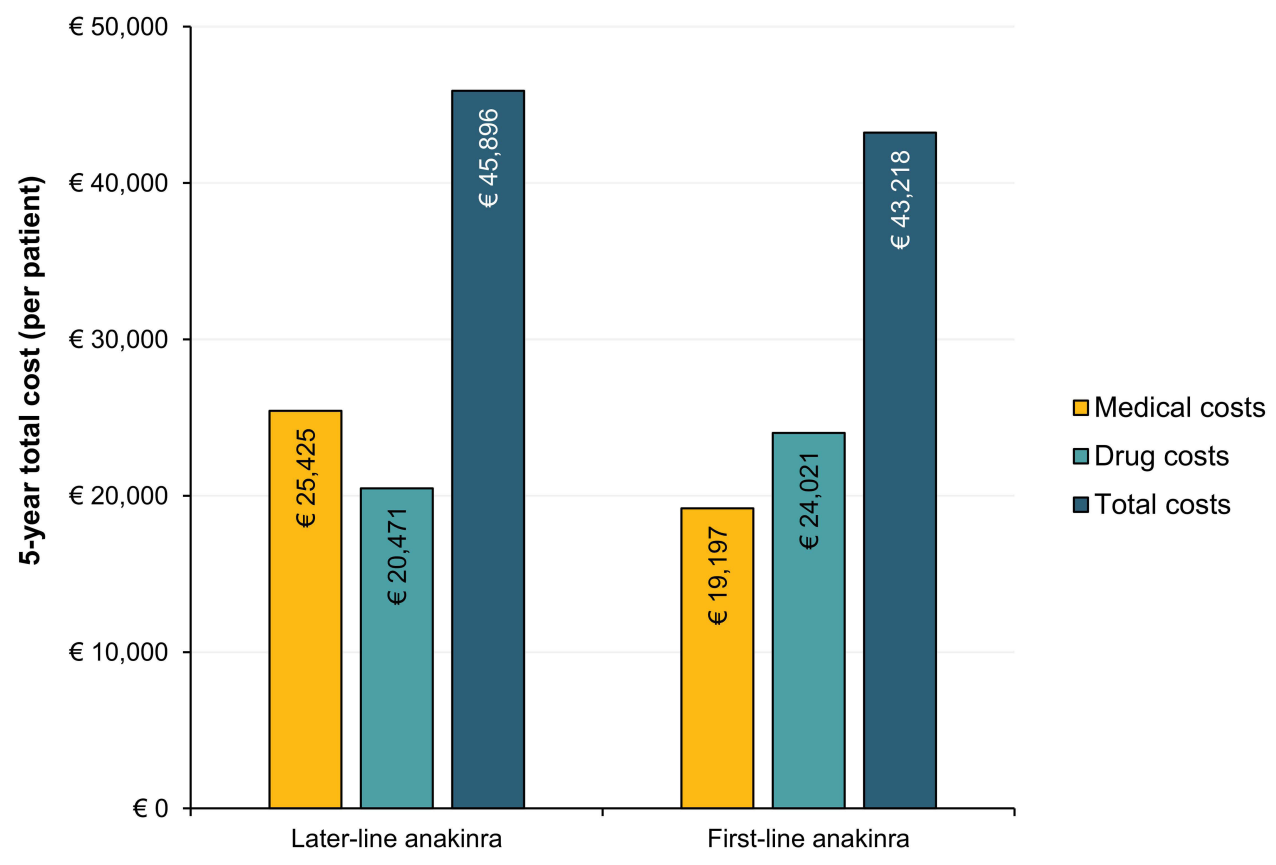

Strategy

Figure I Base-case analysis results. 
cost-saving relative to later-line use, which, when considered in conjunction with the improved clinical outcomes, gives further justification for the use of anakinra earlier in the course of sJIA.

The conclusion reached by the analysis presented may be surprising when considering the costs of biologic therapies in other rheumatic diseases. However, it is important to acknowledge that anakinra was first granted a marketing authorization in March 2002 (for its use in patients with rheumatoid arthritis), and has been used in clinical practice in the management of Still's disease for over a decade. ${ }^{20}$ As such, the weekly cost of anakinra ( $€ 225$, based on Dutch reference costs, last updated June 2020) is relatively low compared with other, more recent bDMARDs, such as canakinumab (Ilaris ${ }^{\circledR}$, Novartis), which has an equivalent weekly cost of approximately $€ 2750$ (assuming one vial is used every 4 weeks, also last updated June 2020).

The clinical evidence in support of using anakinra earlier in the disease course is increasing over time. The SHARE guidelines advocate the use of anakinra as early as possible in sJIA to capitalize on the potential benefits of early IL-1 inhibition. ${ }^{11}$ A recent study by Pardeo et al also demonstrated the probability of achieving CID with anakinra based on the disease duration from onset to start of anakinra $-91.9 \%$ of patients that started anakinra within 3 months of disease onset achieved CID off corticosteroids, versus $36.8 \%$ who started after 3 months of disease onset. $^{25}$ Accordingly, our study provides a timely assessment of the economic implications of earlier versus later use of anakinra.

In addition to anakinra, two other bDMARDs are licensed for the treatment of sJIA: tocilizumab (an IL-6 inhibitor) and canakinumab (an IL-1 $\beta$ inhibitor). However, unlike anakinra, both of these bDMARDs are only licensed for use after previous therapy with NSAIDs and corticosteroids, and tocilizumab is not licensed for the treatment of AOSD. Anakinra may be used within its licensed indication in patients with active systemic features of moderate to high disease activity without prior use of NSAIDs and corticosteroids. This distinction in the marketing authorization for bDMARDs is especially important in consideration of the emerging evidence for the role of targeted therapies in Still's disease, where anakinra can be started as soon as practically possible following diagnosis.

Economic analyses for sJIA treatments are limited in number, which is unsurprising given the rarity of sJIA. Our analysis makes use of some information reported in NICE TA238 (tocilizumab in sJIA). ${ }^{30}$ In addition to the previous NICE assessment of tocilizumab, Kittiratchakool et al recently undertook a cost-effectiveness analysis of tocilizumab in refractory sJIA. ${ }^{38}$ This analysis only considers the potential use of tocilizumab in a relapsed setting, due to its availability in Thailand specifically in this setting. Conversely, our comparison considers the use of bDMARD therapy either in a refractory setting (ie, after NSAID, corticosteroid, and csDMARD treatment) or in a first-line setting - a comparison that (to our knowledge) has not been previously attempted.

We considered a synthesis of available information to compare the alternative treatment strategies. In doing so, we aimed to use the best available evidence to explore the differences in costs for each strategy, allowing for sensitivity analyses to be conducted concerning the key input parameters and associated assumptions. The ability to explore a range of sensitivity analyses for key settings and assumptions is an important advantage of our analytical approach, given that data available to quantify specific assumptions are either limited or in some cases not available (for example, the proportion of patients that achieve sustained CID with csDMARDs in an sJIA population).

Key uncertainties relating to lack of comparative data may be resolved with further evidence collection, though will unavoidably still be subject to various caveats related to differences in practices by geography and difficulties in enrolling patients within trials of treatments that are already routinely available. In particular, the first-line anakinra strategy considered in our economic analysis is based on data from a single-center study by ter Haar et al, though findings could have been different had the study been repeated in other settings or potentially within the context of a multi-center study. Therefore, while our study makes use of several assumptions to populate these missing data, further research and data collection are required to obtain a more robust estimate of the true costs associated with the management of sJIA.

In addition to the limited data available to inform our economic analysis, it is important to acknowledge that sJIA is a heterogeneous disease, and response to treatment may differ based on various patient characteristics. ${ }^{24,39,40}$ These include time since diagnosis, disease subtype or severity, and treatment history. ${ }^{24,39-43}$ Should additional data become available in the future, consideration of how outcomes compare across different patient subpopulations may be of interest from both a clinical and economic perspective. 
The full benefits of first-line anakinra are not fully captured within our analysis, mainly due to the limitations of currently-available evidence. Benefits not captured within the main analysis include a reduced risk of developing MAS, the avoidance of long-run consequences relating to corticosteroid and/or csDMARD exposure, and productivity gains resulting from patients achieving CID. While we attempted to address these in part through sensitivity analysis, these potential benefits should also be considered when interpreting the results of our analysis. The findings presented within this study may be considered a conservative estimate of the potential cost savings associated with first-line anakinra.

\section{Conclusions}

To our knowledge, this study presents the first economic comparison of treatment strategies in sJIA, based on current treatment pathways across Europe. There have been no published studies of medical management costs in Still's disease, based on whether patients have achieved CID and/or which treatment(s) they are currently managed with. The findings of the economic comparison illustrate the value of first-line anakinra in SJIA, when considered in the context of the full costs of patient management.

\section{Abbreviations}

AOSD, Adult-onset Still's disease; bDMARD, Biologic disease-modifying anti-rheumatic drug; $\mathrm{CAD}$, Clinically-active disease; CID, Clinically-inactive disease; csDMARD, Conventional-synthetic disease-modifying anti-rheumatic drug; GP, General practitioner; IL-1/IL-6, Interleukin-1/-6; JIA, Juvenile idiopathic arthritis; MAS, Macrophage activation syndrome; MRU, Medical resource use; NICE, National Institute for Health and Care Excellence; NSAID, Nonsteroidal anti-inflammatory drug; NVK, Nederlandse Vereniging voor Kindergeneeskunde [Dutch Pediatrics Association]; SHARE, Single Hub and Access point for pediatric Rheumatology in Europe; sJIA, Systemic juvenile idiopathic arthritis.

\section{Acknowledgments}

The authors thank a number of practicing clinicians who provided supporting information and opinion referred to within this study. The authors also wish to thank Annelies van Leeuwen-Gorter for her scientific input. Early findings reported in this paper were presented at the Virtual ISPOR Europe 2020 conference as a poster presentation. The poster's abstract was published in volume 23 , supplement 2 of the
Value in Health journal, available at: https://doi.org/10.1016/ j.jval.2020.08.803

\section{Funding}

This study was funded by Swedish Orphan Biovitrum AB (publ), Stockholm, Sweden. Sobi is the marketing authorization holder for anakinra $\left(\right.$ Kineret $\left.^{\circledR}\right)$.

\section{Disclosure}

ML, GRD, JN, and DE are employees of Sobi. AB and EK are employees of Delta Hat Limited, which received funding from Sobi to carry out the economic analysis described here within. The authors report no other conflicts of interest in this work.

\section{References}

1. Modesto C, Antón J, Rodriguez B, et al. Incidence and prevalence of juvenile idiopathic arthritis in Catalonia (Spain). Scand J Rheumatol. 2010;39(6):472-479. doi:10.3109/03009741003742722

2. Pruunsild C, Uibo K, Liivamägi H, Tarraste S, Talvik T, Pelkonen P. Incidence of juvenile idiopathic arthritis in children in Estonia: a prospective population-based study. Scand J Rheumatol. 2007;36 (1):7-13. doi:10.1080/03009740601089259

3. Berntson L, Andersson Gäre B, Fasth A, et al. Incidence of juvenile idiopathic arthritis in the Nordic countries. A population based study with special reference to the validity of the ILAR and EULAR criteria. J Rheumatol. 2003;30(10):2275-2282.

4. Thierry S, Fautrel B, Lemelle I, Guillemin F. Prevalence and incidence of juvenile idiopathic arthritis: a systematic review. Joint Bone Spine. 2014;81(2):112-117. doi:10.1016/j. jbspin.2013.09.003

5. Spiegel LR, Schneider R, Lang BA, et al. Early predictors of poor functional outcome in systemic-onset juvenile rheumatoid arthritis: a multicenter cohort study. Arthritis Rheum off J Am Coll Rheumatol. 2000;43(11):2402-2409. doi:10.1002/1529-0131(200011)43:11<24 02::AID-ANR5>3.0.CO;2-C

6. Bowyer SL, Roettcher PA, Higgins GC, et al. Health status of patients with juvenile rheumatoid arthritis at 1 and 5 years after diagnosis. J Rheumatol. 2003;30(2):394-400.

7. Svantesson H, Akesson A, Eberhardt K, Elborgh R. Prognosis in juvenile rheumatoid arthritis with systemic onset a follow-up study. Scand J Rheumatol. 1983;12(2):139-144. doi:10.3109/030097483 09102900

8. Deslandre J. Systemic-onset juvenile idiopathic arthritis; 2007. Available from: https://www.orpha.net/consor/cgi-bin/Disease_ Search.php?lng=EN\&data_id=11711\&Disease_Disease_Search_ diseaseGroup=Still-disease $\&$ Disease_Disease_Search_diseaseType $=$ Pat\&Disease $(\mathrm{s}) /$ group $\% 20$ of $\% 20$ diseases $=$ Systemic-onset-juvenile -idiopathic-arthritis\&title=Systemic-onset $\% 20$ juvenile $\% 20$ idio pathic\%20arthritis\&search=Disease_Search_Simple. Accessed April 14, 2019.

9. Modica RF, Lomax KG, Batzel P, Cassanas A. Impact of systemic juvenile idiopathic arthritis/Still's disease on adolescents as evidenced through social media posts. Open Access Rheumatol Res Rev. 2018;10:73-81.

10. Bruck N, Schnabel A, Hedrich CM. Current understanding of the pathophysiology of systemic juvenile idiopathic arthritis (sJIA) and target-directed therapeutic approaches. Clin Immunol. 2015;159 (1):72-83. doi:10.1016/j.clim.2015.04.018 
11. Leek AP, Anton J, Avcin T, et al. The SHARE recommendations on diagnosis and treatment of systemic JIA; 2020. Available from: https://acrabstracts.org/abstract/the-share-recommendations-ondiagnosis-and-treatment-of-systemic-jia/. Accessed October 28, 2020 .

12. Bracaglia C, Prencipe G, De Benedetti F. Macrophage activation syndrome: different mechanisms leading to a one clinical syndrome. Pediatr Rheumatol Online J. 2017;15(1):5. doi:10.1186/s12969-0160130-4

13. Ruscitti P, Rago C, Breda L, et al. Macrophage activation syndrome in Still's disease: analysis of clinical characteristics and survival in paediatric and adult patients. Clin Rheumatol. 2017;36 (12):2839-2845. doi:10.1007/s10067-017-3830-3

14. Woo P, Southwood TR, Prieur AM, et al. Randomized, placebo-controlled, crossover trial of low-dose oral methotrexate in children with extended oligoarticular or systemic arthritis. Arthritis Rheum. 2000;43(8):1849-1857. doi:10.1002/1529-0131(200008) 43:8<1849::AID-ANR22>3.0.CO;2-F

15. Quartier P, Allantaz F, Cimaz R, et al. A multicentre, randomised, double-blind, placebo-controlled trial with the interleukin-1 receptor antagonist anakinra in patients with systemic-onset juvenile idiopathic arthritis (ANAJIS trial). Ann Rheum Dis. 2011;70 (5):747-754. doi:10.1136/ard.2010.134254

16. Ilowite N, Porras O, Reiff A, et al. Anakinra in the treatment of polyarticular-course juvenile rheumatoid arthritis: safety and preliminary efficacy results of a randomized multicenter study. Clin Rheumatol. 2009;28(2):129-137. doi:10.1007/s10067-008-0995-9

17. Nordström D, Knight A, Luukkainen R, et al. Beneficial effect of interleukin 1 inhibition with anakinra in adult-onset Still's disease. An open, randomized, multicenter study. J Rheumatol. 2012;39 (10):2008-2011. doi:10.3899/jrheum.111549

18. Verbsky JW, White AJ. Effective use of the recombinant interleukin 1 receptor antagonist anakinra in therapy resistant systemic onset juvenile rheumatoid arthritis. $J$ Rheumatol. 2004;31 (10):2071-2075.

19. De Benedetti F, Brunner HI, Ruperto N, et al. Randomized trial of tocilizumab in systemic juvenile idiopathic arthritis. $N$ Engl J Med. 2012;367(25):2385-2395. doi:10.1056/NEJMoa1112802

20. Swedish Orphan Biovitrum AB. Summary of product characteristics: anakinra (Kineret); 2020. Available from: https://www.ema.europa. eu/en/documents/product-information/kineret-epar-productinformation_en.pdf. Accessed August 17, 2021.

21. Ter Haar NM, van Dijkhuizen EHP, Swart JF, et al. Treatment to target using recombinant interleukin-1 receptor antagonist as first-line monotherapy in new-onset systemic juvenile idiopathic arthritis: results from a five-year follow-up study. Arthritis Rheumatol Hoboken NJ. 2019;71(7):1163-1173. doi:10.1002/art.40865

22. Vastert SJ, de Jager W, Noordman BJ, et al. Effectiveness of first-line treatment with recombinant interleukin-1 receptor antagonist in steroid-naive patients with new-onset systemic juvenile idiopathic arthritis: results of a prospective cohort study. Arthritis Rheumatol. 2014;66(4):1034-1043. doi:10.1002/art.38296

23. Rossi MN, Pardeo M, Marafon DP, Sacco E, Passarelli C. Early treatment and IL1RN single nucleotide polymorphisms affect response to anakinra in systemic juvenile idiopathic arthritis. ACR Meeting Abstracts; 2019. Available from: https://acrabstracts.org/ abstract/early-treatment-and-il1rn-single-nucleotide-polymorphismsaffect-response-to-anakinra-in-systemic-juvenile-idiopathic-arthritis/. Accessed October 28, 2020.

24. Pardeo M, Pires Marafon D, Insalaco A, et al. Anakinra in systemic juvenile idiopathic arthritis: a single-center experience. J Rheumatol. 2015;42(8):1523-1527. doi:10.3899/jrheum.141567

25. Pardeo M, Rossi MN, Marafon DP, et al. Early treatment and IL1RN SNPs affect response to anakinra in systemic juvenile idiopathic arthritis - author copy provided pre-publication; 2020.
26. Richtlijn NVK. Juveniele idiopathische artritis, medicamenteuze behandeling van kinderen met; 2018. Available from: https:/www.nvk.nl/ themas/kwaliteit/richtlijnen/richtlijn?componentid=7864339\&tagtitles= Infectieziekten+en+Immunologie,Intensive+Care,Maag-DarmLeverziekten+(MDL), Reumatologie,Sociale+en+Psychosociale+kinder geneeskunde,Chirurgie. Accessed April 14, 2021.

27. Schanberg L, Nigrovic P, Cooper A, et al., Double-blind, placebocontrolled study of anakinra in pediatric and adult patients with still's disease. ACR Meeting Abstracts; 2020. Available from: https://acrab stracts.org/abstract/a-randomized-double-blind-placebo-controlled-studyof-anakinra-in-pediatric-and-adult-patients-with-stills-disease/. Accessed April 14, 2021.

28. Nigrovic PA. Review: is there a window of opportunity for treatment of systemic juvenile idiopathic arthritis? Arthritis Rheumatol. 2014;66(6):1405-1413. doi:10.1002/art.38615

29. Pardeo M, Bracaglia C, Tulone A, et al. Early treatment with anakinra in systemic juvenile idiopathic arthritis. ACR Meeting Abstracts; 2019. Available from: https://acrabstracts.org/abstract/ early-treatment-with-anakinra-in-systemic-juvenile-idiopathicarthritis/. Accessed February 5, 2020.

30. National Institute for Health and Care Excellence (NICE). Tocilizumab for the treatment of systemic juvenile idiopathic arthritis: technology appraisal guidance [TA238]; 2011. Available from: https://www.nice.org.uk/guidance/ta238. Accessed April 14, 2019.

31. National Institute for Health and Care Excellence (NICE). Anakinra for treating Still's disease: technology appraisal guidance [TA685]; 2021. Available from: https://www.nice.org.uk/guidance/ta685. Accessed April 14, 2021.

32. England NHS. Clinical commissioning policy statement: biologic therapies for the treatment of juvenile idiopathic arthritis (JIA) (E03X04). London: NHS England; 2015. Available from: https:// www.england.nhs.uk/commissioning/wp-content/uploads/sites/12/ 2015/10/e03pd-bio-therapies-jia-oct15.pdf. Accessed August 17, 2021.

33. Grevich S, Shenoi S. Update on the management of systemic juvenile idiopathic arthritis and role of IL-1 and IL-6 inhibition. Adolesc Health Med Ther. 2017;8:125.

34. Hakkaart-van Roijen L, Van der Linden N, Bouwmans C, Kanters T, Tann S Costing manual: methodology of costing research and reference prices for economic evaluations in healthcare; 2015. Available from: https://www.imta.nl/costingtool/. Accessed October 31, 2020.

35. Z-Index BV. G-Standaard, the Dutch drug database; 2020. Available from: https://www.z-index.nl/english. Accessed June 19, 2020.

36. Grom AA, Ilowite NT, Pascual V, et al. Rate and clinical presentation of macrophage activation syndrome in patients with systemic juvenile idiopathic arthritis treated with canakinumab. Arthritis Rheumatol. 2016;68(1):218-228. doi:10.1002/art.39407

37. Ferrara G, Petrillo MG, Giani T, et al. Clinical use and molecular action of corticosteroids in the pediatric age. Int J Mol Sci. 2019;20 (2):444. doi:10.3390/ijms20020444

38. Kittiratchakool N, Kulpokin D, Chanjam C, et al. Cost-utility and budget impact analysis of tocilizumab for the treatment of refractory systemic juvenile idiopathic arthritis in Thailand. BMJ Open. 2020;10 (9):e037588. doi:10.1136/bmjopen-2020-037588

39. Boyarchuk O, Kovalchuk T, Kovalchuk N, Chubata O. Clinical variability of the systemic juvenile idiopathic arthritis course: literature review based on case series. Reumatologia. 2020;58(6):436-443. doi:10.5114/reum.2020.102010

40. Giancane G, Minoia F, Davì S, Bracciolini G, Consolaro A, Ravelli A. IL-1 inhibition in systemic juvenile idiopathic arthritis. Front Pharmacol. 2016;7:467. doi:10.3389/fphar.2016.00467

41. Vastert SJ, Jamilloux Y, Quartier P, et al. Anakinra in children and adults with Still's disease. Rheumatology. 2019;58(Supplement_6): vi9-22. doi:10.1093/rheumatology/kez350 
42. Nigrovic PA, Mannion M, Prince FH, et al. Anakinra as first-line disease-modifying therapy in systemic juvenile idiopathic arthritis: report of forty-six patients from an international multicenter series. Arthritis Rheum. 2011;63(2):545-555. doi:10.1002/art.30128
43. Woerner A, Uettwiller F, Melki I, et al. Biological treatment in systemic juvenile idiopathic arthritis: achievement of inactive disease or clinical remission on a first, second or third biological agent. $R M D$ Open. 2015;1(1):e000036. doi:10.1136/rmdopen-2014-000036

\section{Publish your work in this journal}

Open Access Rheumatology Research and Reviews is an international, peer-reviewed, open access journal publishing original research, reports, editorials, reviews and commentaries on all aspects of clinical and experimental rheumatology in the clinic and laboratory including the following topics: Pathology, pathophysiology of rheumatological diseases; Investigation, treatment and management of rheumatological diseases; Clinical trials and novel pharmacological approaches for the treatment of rheumatological disorders. The manuscript management system is completely online and includes a very quick and fair peer-review system, which is all easy to use. Visit http://www.dovepress.com/testimonials.php to read real quotes from published authors. 